\title{
Osteoartrite global do cotovelo após uma fratura não deslocada da cabeça radial: Relato de caso*
}

\section{Severe Osteoarthritis of the Elbow after a Non-Displaced Radial Head Fracture: Case Report}

\author{
Lauro Augusto Veloso Costa ${ }^{1}$ Bruno Cesar Pereira Brabo ${ }^{1,2}$ André Luís Marangoni ${ }^{2}$ \\ Luiz Fabiano Presente Taniguchi ${ }^{1}$ \\ ${ }^{1}$ Hospital Municipal Dr. Moysés Deutsch, São Paulo, SP, Brasil \\ 2 Hospital Municipal Dr. Carmino Caricchio, São Paulo, SP, Brasil \\ Rev Bras Ortop 2020;55(3):383-385. \\ Endereço para correspondência Lauro Augusto Veloso Costa, MD, \\ MSc, Hospital Municipal Dr. Moysés Deutsch; Setor de Ortopedia e \\ Traumatologia, Estrada do M’boi Mirim, n 5203, Jardim Ângela, \\ São Paulo, SP, Brasil (e-mail: lauroavc@hotmail.com).
}

\section{Resumo \\ Palavras-chave \\ - cotovelo \\ - osteoartrite \\ - fraturas do rádio}

As fraturas da cabeça radial representam mais de $50 \%$ de todas as fraturas do antebraço. Broberg e Morrey (modificação da classificação de Mason) as classificam em quatro tipos. As fraturas do tipo 1 são aquelas não deslocadas ou minimamente deslocadas, consideradas estáveis e sem bloqueio mecânico das articulações. Nesse tipo de fratura, um manejo não operatório com um curto período de imobilização permite bom resultado clínico sem desfechos adversos. Lesões associadas clinicamente relevantes não são comuns. Por esse motivo, a artrite da articulação radiocapitelar e ulnoumeral após a fratura da cabeça do rádio não deslocada é incomum. Este relato de caso apresenta um paciente jovem com diagnóstico de fratura isolada da cabeça radial não deslocada que evoluiu, 8 meses depois, para artrite global do cotovelo.

Fractures of the radial head account for over $50 \%$ of all fractures of the forearm. Broberg and Morrey (modification of the Mason classification) classify them in four types. Type 1 fractures are non-displaced or minimally displaced, considered stable and without mechanical joint blockage. In this type of fracture, a non-operative management with a short period of immobilization leads to a good clinical result without adverse outcomes. Clinically relevant associated lesions are not common. Because of this, arthritis of the radiocapitellar and ulnohumeral joint after the non-displaced radial head fracture is uncommon. This case report presents a young patient diagnosed with isolated non-displaced radial head fracture, that evolved 8 months later, to global arthritis of the elbow.

\section{Introdução}

As fraturas da cabeça radial representam mais de $50 \%$ de todas as fraturas do antebraço. Essas fraturas ocorrem com

\footnotetext{
Trabalho desenvolvido no Hospital Municipal Dr. Moysés
} Deutsch, São Paulo, SP, Brasil.

recebido

14 de Agosto de 2019

aceito

03 de Outubro de 2019
DOI https://doi.org/

10.1055/s-0039-3402468. ISSN 0102-3616. um trauma indireto através do longo eixo do rádio durante uma queda com o antebraço pronado e parcialmente flexionado. ${ }^{1,2}$ Broberg e Morrey as classificam em quatro tipos (modificação da classificação Mason). As fraturas do tipo 1 são aquelas não deslocadas ou minimamente deslocadas (deslocadas em não mais de $2 \mathrm{~mm}$ ) e o tamanho do fragmento deve incluir até 30\% da cabeça (superfície articular).
Copyright $\odot 2020$ by Sociedade Brasileira License terms de Ortopedia e Traumatologia. Published by Thieme Revinter Publicações Ltda, Rio de Janeiro, Brazil 
Se a fratura é deslocada em $2 \mathrm{~mm}$ ou mais, ou envolve 30\% da cabeça, é considerada o tipo 2. Do tipo 3 são as fraturas cominutivas. Finalmente, fraturas do tipo 4 são as aquelas com luxação do cotovelo. ${ }^{2,3}$

As fraturas do tipo 1 são causadas por trauma de baixa energia. Correspondem de 64 a $74 \%$ de todas as fraturas da cabeça radial. ${ }^{1,4}$ Levando em conta suas características, a fratura do tipo 1 é considerada estável e não apresenta bloqueio articular mecânico. Além disso, lesões associadas clinicamente relevantes não são comuns. ${ }^{5}$ Por tudo isso, nesse tipo de fratura, um manejo não operatório, com um curto período de imobilização, permite um bom resultado clínico sem desfechos adversos. ${ }^{4}$ A osteoartrite da radiocapitelar (OA RC) e da articulação ulnoumeral é incomum após a fratura da cabeça radial não deslocada. ${ }^{6}$

Este relato de caso apresenta um paciente jovem com diagnóstico de fratura isolada e não deslocada da cabeça radial que evoluiu 8 meses depois com artrite global do cotovelo. Por meio de revisão sobre fratura da cabeça radial não deslocada, este artigo destaca a importância do acompanhamento dos pacientes para reconhecer essa possível complicação, mesmo em lesões consideradas simples.

\section{Relato de Caso}

Um homem de 35 anos, porteiro, caiu durante um jogo de futebol. Após 3 dias da lesão, o paciente compareceu ao hospital para atendimento médico. No exame físico apresentava um inchaço comum no cotovelo e sensibilidade sobre a cabeça radial. A estabilidade do cotovelo foi verificada e não havia suspeita de lesão ligamentar. Após radiografia e tomografia computadorizada, o paciente foi diagnosticado com fratura de cabeça radial não deslocada (-Fig. 1a, b e -Fig. 2a, b).

O tratamento inicial foi imobilização de todo o braço com gesso. Como o paciente não sentia mais dor após 7 dias de imobilização, ele parou de usar o gesso e voltou ao trabalho. Dois meses depois, o paciente realizou nova radiografia que mostrou consolidação com pouca deformidade da cabeça radial e baixo grau de osteoartrose ( - Fig. 3a, b). No entanto, apenas 6 meses depois, o paciente veio até nós com queixas de dor e limitação de movimento. Durante o exame físico, o paciente apresentou amplitude de movimento reduzida ($80^{\circ}$ de extensão e $110^{\circ}$ de flexão), mas não apresentou limitação importante na rotação do antebraço. Em seguida,

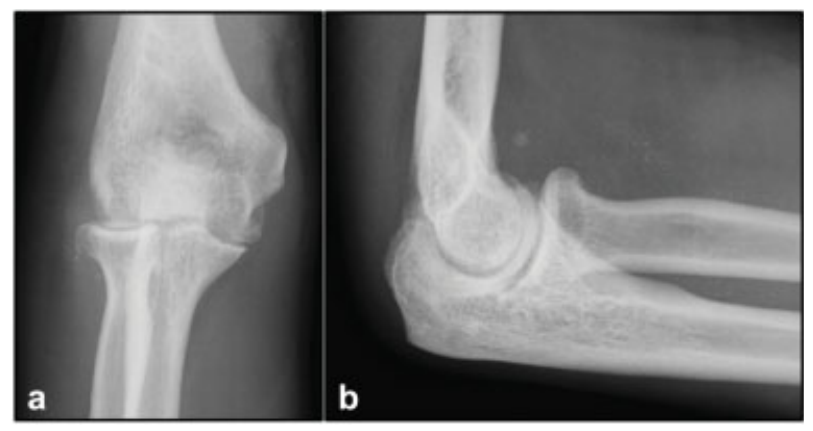

Fig. 1 Radiografias iniciais do cotovelo: visões anteroposterior (a) e lateral (b).
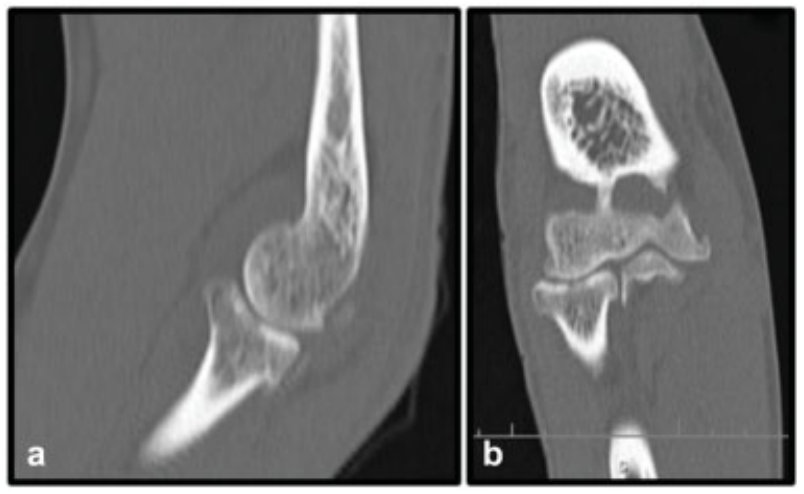

Fig. 2 A tomografia computadorizada mostrou fratura da cabeça radial não deslocada e isolada: visões sagital (a) e coronal (b).

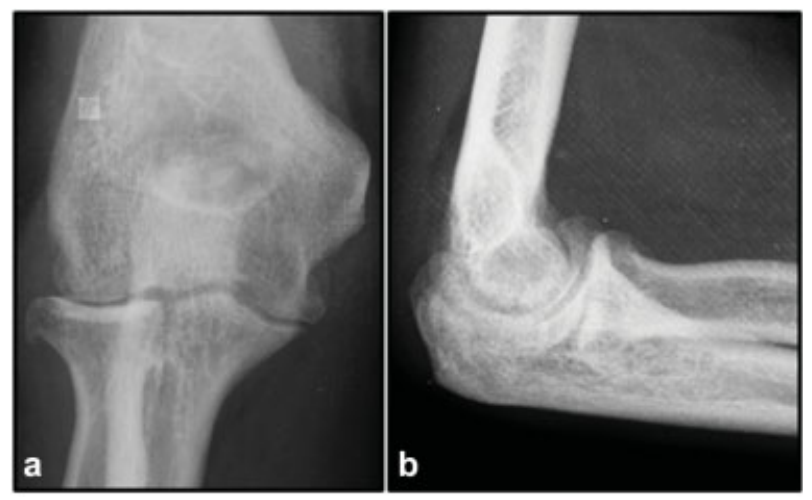

Fig. 3 Radiografias após 2 meses da lesão demonstrando consolidação de fratura com pouca deformidade da cabeça radial e baixo grau de osteoartrose: visões anteroposterior (a) e lateral (b).

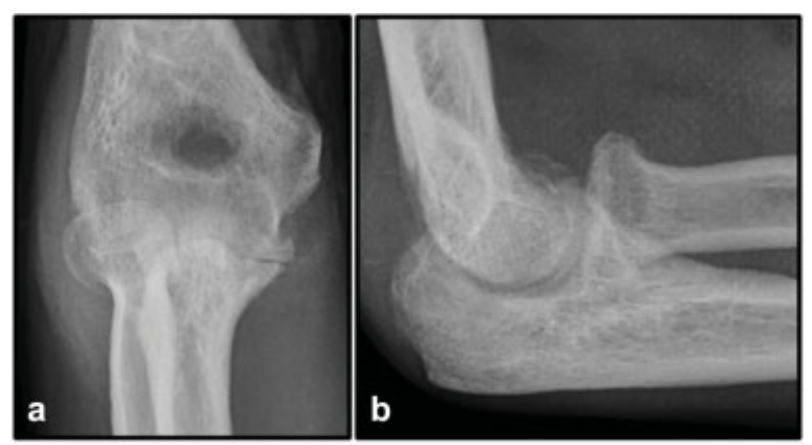

Fig. 4 Visões anteroposterior (a) e lateral (b) do cotovelo 8 meses após a lesão inicial, mostrando osteoartrite global e grave.

o paciente realizou novas radiografias do cotovelo que evidenciavam osteoartrite grave do cotovelo ( - Fig. 4 a, b). Em seguida, o paciente foi submetido ao desbridamento artroscópico do cotovelo, com importante melhora na amplitude de movimento e dor.

\section{Discussão}

Os principais resultados adversos relacionados à fratura radial da cabeça não deslocada são rigidez do cotovelo e 
dor ocasional no cotovelo. ${ }^{4,5,7}$ A artrose do cotovelo após esse tipo de fratura é rara. ${ }^{6}$

Herbertsson et al. ${ }^{7}$ avaliaram os resultados a longo prazo de 27 fraturas de Mason tipo 1 e relataram que, embora os cotovelos lesionados tenham mais alterações degenerativas do que os cotovelos não lesionados, esses achados não têm significado clínico. Os casos, em sua maioria, estavam livres de dor e não apresentavam perda de movimento. Eles também relataram que os cotovelos lesionados tinham mais deformidades radiológicas do que os cotovelos não lesionados, mas essas deformidades não estão associadas à osteoartrite. Kachooei et al. ${ }^{6}$ avaliaram se pacientes após tratamento não operatório de uma fratura isolada da cabeça do rádio tinham $\mathrm{OA} \mathrm{RC}$. Dos 54 pacientes avaliados, 1 apresentava OA RC isolado e 3 também tinham osteoartrite ulnoumeral. Concluíram, portanto, que a OA RC é incomum após essas fraturas, mesmo com algum grau de deformidade da cabeça radial.

A estabilidade do cotovelo deve ser verificada no exame físico inicial para avaliar lesões ligamentares, pois a fratura da cabeça radial pode fazer parte de uma lesão complexa do cotovelo. Kaas et al. $^{5}$ relataram lesões associadas usando ressonância magnética (RM) em 12 de 17 pacientes com fratura Mason tipo 1. No seu estudo, demonstraram que $47 \%$ dos pacientes diagnosticados com esse tipo de fratura também apresentavam lesão do ligamento colateral lateral (LCL) e 2 de 17 pacientes apresentaram lesão condral. Destes pacientes, 7 tinham uma ruptura parcial e um tinha uma ruptura completa. Embora essas lesões sejam visíveis na ressonância magnética, elas raramente são sintomáticas. Além da lesão do LCL, as lesões da membrana interóssea (MIO) também são comumente associadas com fraturas do tipo Mason 1. Hausmann et al. ${ }^{8}$ examinaram 14 pacientes com fratura de Mason tipo 1 quanto a lesão oculta na MIO. Eles relataram que 9 dos 14 pacientes apresentavam lesões adicionais da parte distal da MIO. Como os pacientes podem ter lesões adicionais, é provável que a osteoartrite do cotovelo após a fratura do tipo Mason 1 tenha sido parte de uma lesão ligamentar mais complexa.

No nosso caso apresentado, o paciente foi diagnosticado com artrose pós-traumática grave. No entanto, no exame físico inicial, não suspeitamos de instabilidade derivada de lesão ligamentar. Portanto, como o paciente sofreu um trauma de alta energia, é provável que ele tenha tido uma lesão condral não diagnosticada pelas imagens. Outra hipó- tese é que o paciente tivesse uma lesão ligamentar não diagnosticada no exame físico inicial, uma vez que o exame para o diagnóstico de uma lesão sutil no ligamento do cotovelo não é fácil de reproduzir. Em ambas as hipóteses, uma RM do cotovelo provavelmente teria ajudado nesse diagnóstico e, portanto, pode ser necessária sempre que houver um trauma de alta energia.

Em conclusão, fraturas da cabeça radial do tipo Mason 1 são fraturas com baixo risco de desenvolver osteoartrite após tratamento conservador. No entanto, não há garantia de resultado favorável. Sempre que houver fortes suspeitas clínicas de lesões mais complexas, como no trauma de alta energia, uma RM pode ser solicitada, e o paciente deve ser acompanhado para que qualquer possível complicação seja identificada mais cedo.

Conflito de Interesses

Os autores declaram não haver conflito de interesses.

\section{Referências}

1 Mahmoud SS, Moideen AN, Kotwal R, Mohanty K. Management of Mason type 1 radial head fractures: a regional survey and a review of literature. Eur J Orthop Surg Traumatol 2014;24(07): 1133-1137

2 Mason ML. Some observations on fractures of the head of the radius with a review of one hundred cases. Br J Surg 1954;42 (172):123-132

3 Broberg MA, Morrey BF. Results of treatment of fracture-dislocations of the elbow. Clin Orthop Relat Res 1987;(216):109-119

4 Duckworth AD, McQueen MM, Ring D. Fractures of the radial head. Bone Joint J 2013;95-B(02):151-159

5 Kaas L, van Riet RP, Turkenburg JL, Vroemen JP, van Dijk CN, Eygendaal D. Magnetic resonance imaging in radial head fractures: most associated injuries are not clinically relevant. J Shoulder Elbow Surg 2011;20(08):1282-1288

6 Kachooei AR, Ring D. Evaluation of Radiocapitellar Arthritis in Patients with a Second Radiograph at Least 2 Years after Nonoperative Treatment of an Isolated Radial Head Fracture. Arch Bone Jt Surg 2017;5(06):375-379

7 Herbertsson P, Josefsson PO, Hasserius R, Karlsson C, Besjakov J, Karlsson MK. Displaced Mason type I fractures of the radial head and neck in adults: a fifteen- to thirty-three-year follow-up study. J Shoulder Elbow Surg 2005;14(01):73-77

8 Hausmann JT, Vekszler G, Breitenseher M, Braunsteiner T, Vécsei V, Gäbler C. Mason type-I radial head fractures and interosseous membrane lesions-a prospective study. J Trauma 2009;66(02): 457-461 